\title{
Peak age of information in slotted ALOHA network
}

This paper was downloaded from TechRxiv (https://www.techrxiv.org).

\section{LICENSE}

CC BY 4.0

SUBMISSION DATE / POSTED DATE

08-11-2021/11-11-2021

\section{CITATION}

Asvadi, Sepehr; Ashtiani, Farid (2021): Peak age of information in slotted ALOHA network. TechRxiv. Preprint. https://doi.org/10.36227/techrxiv.16955617.v1

$\mathrm{DOI}$ 


\title{
Peak Age of Information in slotted ALOHA Network
}

\author{
Sepehr Asvadi and Farid Ashtiani \\ Sharif University of technology, Tehran, Iran \\ \{Email: sepehr.asvadi@gmail.com, ashtianimt@ sharif.edu\}
}

\begin{abstract}
In this paper, we concentrate on the peak age of information (PAoI) in a discrete-time slotted ALOHA network comprised of $M$ buffer-less nodes, capable of keeping just one packet at each time. In this network, whenever some nodes transmit their packets simultaneously, a collision happens, thus there is interaction among queues and transmission delay of a packet might prolong more than one slot. The packets are generated at each node stochastically and independently. The nodes follow preemptive queueing policy. In this network, we propose a new trellis-based analytical model to represent the interaction among queues and derive exact average PAoI in closed form. Furthermore, we find average PAoI-constrained regions and minimize average PAoI for a symmetric network, numerically. As a byproduct of our analysis, the achievable throughput region of two slotted ALOHA networks with unlimited buffer and buffer-less nodes are compared. We observe that the latter is superior not only in AoI, but also in achievable throughput region. Simulation results confirm our analysis.
\end{abstract}

Index Terms - Age-of-information (AoI), Peak AoI (PAoI), PAoI-constrained region, preemptive policy, slotted ALOHA.

\section{INTRODUCTION}

Status updating is a crucial task to be done in modern communication networks, e.g., vehicular ad hoc networks, sensor networks, etc. In fact, in these networks, in order to decide correctly, the decision-maker (destination) needs updated status information. Age-of-Information (AoI) is a performance metric, introduced in [1], which considers not only the end-to-end packet delay but 
also the time duration that the destination remains outdated. At any time $t$, if the latest status information (packet), delivered to the destination, was generated at time $U(t)$, then the AoI at the destination is computed as

$$
\Delta(t)=t-U(t)
$$

In [2] the peak AoI (PAoI) is introduced as another related metric that only concentrates on local peaks of $\Delta(t)$.

From a historical point of view, the premier works on AoI considered a communication link comprised of a pair of transmitter-receiver and studied AoI for different queueing policies. Firstcome-first-serve (FCFS) policy was analyzed in [3] for M/M/1, M/D/1, and D/M/1 queues and seemed to be inefficient when packet arrival rate is relatively high. Preemptive and non-preemptive last-come-first-serve (LCFS) policies were investigated in [4] and [5] with exponentially and gamma distributed service times, respectively. The authors in [6] showed the preemptive policy is the optimal policy when service times are distributed exponentially even in multi-server queues. The authors in [7] showed that by using infinite incremental redundancy and finite redundancy hybrid ARQ (HARQ), blocking policy is beneficial over preemptive policy, while the authors in [8] showed in time slotted scenarios equipped with HARQ, each policy could be superior to the other one based on network conditions.

Recently, analysis of AoI in multi-source or multi-hop networks has got attention. AoI in multihop networks was investigated in some works (e.g., [9]-[10]). Scheduled transmissions in broadcast networks was investigated in several works (e.g., [11]-[14]) and Whittle-index policy was shown to be able to achieve near optimal AoI among other scheduling policies in some scenarios.

Different papers studied AoI in some well-known multiple access protocols. The authors in [15] and [16] compared AoI in TDMA and FDMA while the authors in [17] compared AoI in nonorthogonal multiple access (NOMA) and orthogonal multiple access (OMA).

There are several works that analyzed AoI in random access protocols. These works could be 
divided into two main categories. The first category includes the papers with the assumption that packet generation is at will, i.e., a fresh version of information is available at any time ([18]-[22]). The authors in [18] followed a game-theoretical approach for carrier sense multiple access (CSMA) with both AoI-interested and throughput-interested nodes which try to maximize their own utilities. The authors in [19] studied slotted ALOHA-like network and in [20] studied a slotted ALOHA network where each node is allowed to transmit its information if its AoI exceeds a specific threshold. The authors in [21] studied AoI of a lazy version of slotted ALOHA network. The authors in [22] studied AoI under randomized scheduling policies in a wireless communication network.

The second category of works studying AoI in random access protocols includes the papers where the packet generation process is a stochastic process ([23]-[26]). In this category, interaction among the queues is the most challenging obstacle to evaluate AoI. Actually, the communication traffic load that each node sees from the other nodes is a time-varying traffic. Moreover, each node will affect the state of other nodes. For example, if transmissions of two nodes have been collided in a time slot, both nodes would definitely have a packet to transmit in the next slot. Thus, the analysis of each node could not be isolated from the analysis of other nodes. Hence, each node has an interaction with others. Note that the interaction among the queues does not exist in the previous category. Because, when all nodes are always ready to transmit, the traffic load that each node sees from the other nodes is a static one, thus each node would have a collision-free transmission with the same probability at each time slot.

In the aforementioned second category of works, except [23] that only provides some simulation results (without analytical results) on slotted ALOHA network, the exact value of AoI at their scenario has not found and there are different approximations in these works. The authors in [24] found an upper bound on AoI in CSMA environment. To not face the challenge of interacting queues, they considered that all nodes always transmit a packet, no matter have a real one or not. In [25], the authors studied PAoI in slotted ALOHA network by approximately considering that 
each node sees a time invariant traffic from other nodes, i.e., they considered that each node sees the traffic load of other nodes in average. In [26], the authors provided asymptotic results for optimizing AoI in random access channels when the number of sources approaches to infinity.

In this paper, we consider a slotted ALOHA network with erasure channels, comprised of $M$ buffer-less nodes within the coverage of each other and with Bernoulli packet arrival process with rate $\lambda_{i}$ at node $i$. We evaluate the exact average PAoI in this scenario. The most important challenge in this network is due to interaction among queues. As mentioned before, most analytic works on AoI with random access MAC protocols, do not face the challenge of interacting queues, either by limiting their scenario to the case in which packets of each node are available at each slot, or by considering some approximations like that each node sees a time invariant traffic. Although this approximation could be acceptable in some special cases, for example when the number of nodes is relatively high, in general, the difference between PAoIs in the approximated and the real scenarios is not negligible.

The main challenge in our analysis, i.e., interaction among queues, has been considered before in the analytical evaluation of stability region, i.e., achievable throughput region, in a slotted ALOHA network in few cases, e.g., the case of two nodes [27] and three nodes [28]. In [29] an approximate approach to find delay in random access networks has been presented but do not completely model the interaction among the queues. However, since in our scenario the nodes are buffer-less, we propose a trellis-based structure to model the interaction among the queues and use a finite state multi-dimensional discrete time Markov chain (DTMC) to obtain the necessary initial values for the trellis-based model. The importance of this work is that by the proposed method, exact value of PAoI in slotted ALOHA network could be evaluated. Succinctly, our main contributions are as in the following:

- We propose a trellis-based model for dealing with interaction among queues, suitable for the case in which nodes have limited buffer.

- $\quad$ By exploiting the proposed trellis model, we derive average PAoI in a slotted ALOHA network 
analytically.

- By numerical analysis, we find PAoI-constrained region over different parameters, i.e., arrival rates as well as transmission rates, such that average PAoI of different nodes is constrained to be less than some specific thresholds. We will observe that the concerned regions are convex.

- In a symmetric scenario in which all nodes have the same arrival rates and transmission probabilities, we find the optimum PAoI versus the number of nodes and derive the optimum arrival rate and transmission probability, numerically.

- We find achievable throughput region for the case of two nodes and show it includes the corresponding region for the case of nodes with unlimited buffer, i.e., stability region.

The rest of the paper is organized as follows. In Section II, we introduce the network scenario and the main problem we dealt with. In Section III, we find average PAoI in our scenario with two nodes and in Section IV, will generalize our analytical approach to the network with an arbitrary number of nodes. In Section V, we present some numerical results and illustrate PAoI-constrained region in different cases. We also deduce some interesting results for some special cases of network scenarios. Section VI concludes the paper.

\section{Network SCENARIO AND PROBLEM DESCRIPTION}

The network considered in this paper is a slotted ALOHA network (similar to [19]) comprised of a destination and $M$ source nodes as shown in Fig. 1. The time is slotted and at source node $i$, status updates (i.e., new packets) are independently arrived (generated) at each slot with probability $\lambda_{i}$ (i.e., Bernoulli process). Since each node is able to keep only one packet, it is considered as a buffer-less node with just a server. Regarding the importance of freshness of the information, the preemption in service policy ([30]) has been assumed, so the server keeps only the last generated packet. This packet management policy is considered since transmitting the freshest available packet is obviously age-beneficial if residual transmission time of an old packet has the same distribution as the transmission time of a new packet. Since in our scenario, the 


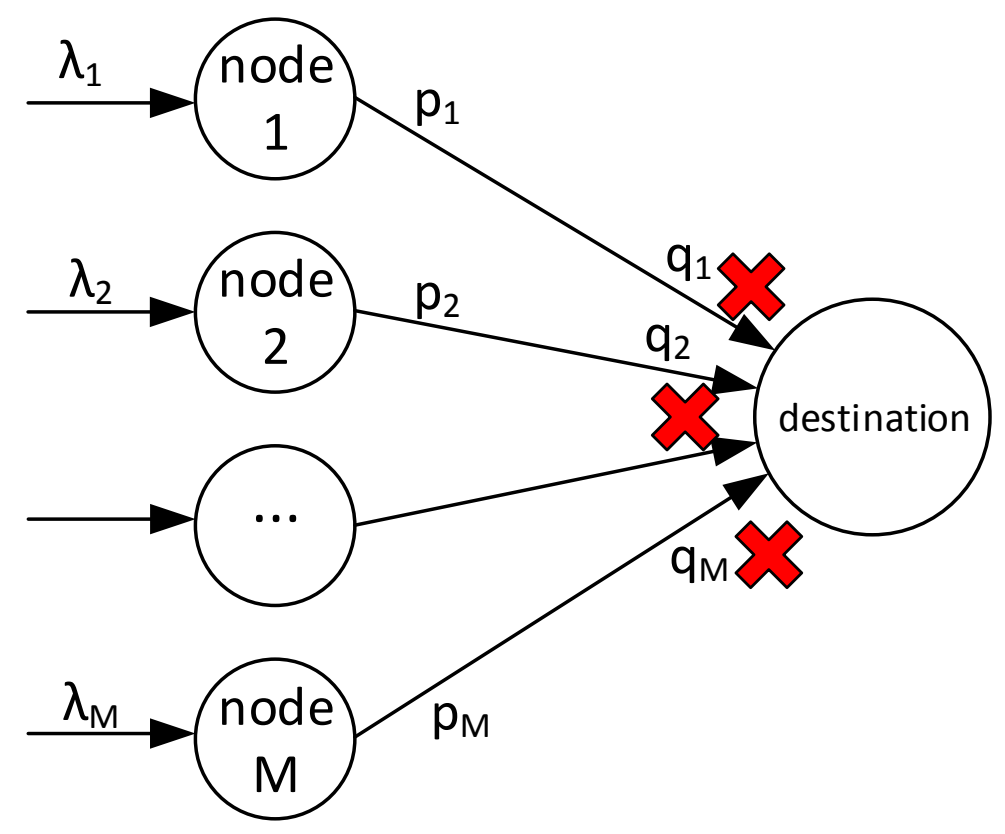

Fig. 1. Structure of the investigating network.

channels are assumed to be independent erasure channels, this condition is satisfied in our work, so we adopt the preemptive policy. At each slot, all nodes follow slotted ALOHA protocol, i.e., if node $i$ has a packet, it will transmit its packet with probability $p_{i}$. We also consider early departure-late arrival (ED-LA) convention in which the transmission (departure) of a packet in a typical time slot occurs earlier than the arrival of a new packet at the same slot (see Fig. 2). Thus, a newly arrived packet cannot be transmitted at the same slot of its arrival. Each packet transmission takes one slot but transmission of a packet could be unsuccessful for two reasons. First, whenever some nodes transmit their packets at the same slot, a collision happens, thus the packets need to be retransmitted. Second, if in a time slot only source $i$ transmits its packet and the other nodes do not transmit any packet, though the transmission would not face a collision, due to physical channel imperfections, it may be independently erased at the destination with probability $q_{i}$ (see Fig. 1). So, it needs to be retransmitted unless a new packet arrives at the end of this time slot and preempts the older packet. It has been assumed that after each packet transmission the destination sends an acknowledgement (ACK)/negative ACK (NACK) at the end of the same slot 


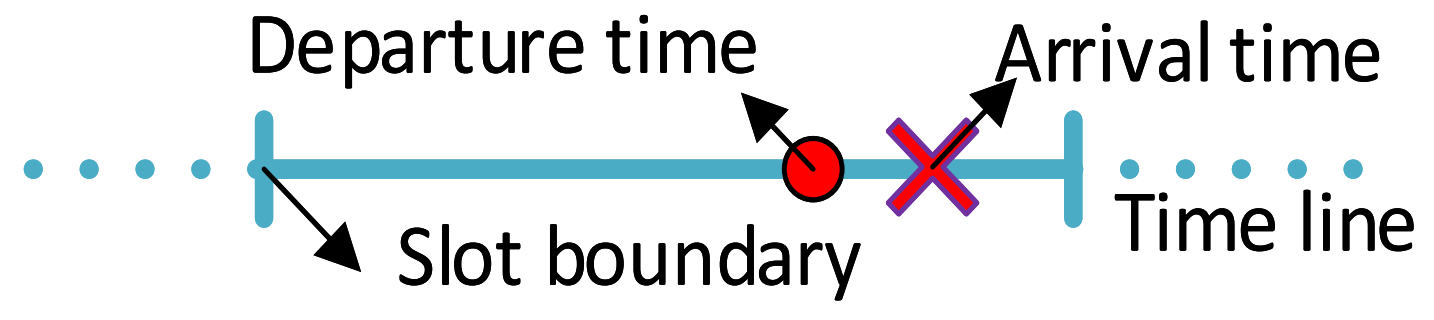

Fig. 2. Early arrival-late departure convection for a typical time slot.

in an error-free channel.

If a packet is received successfully (the transmitter becomes aware by receiving ACK from the destination), it is departed from its corresponding node (server). If a new packet arrives at a node while its server is occupied with another packet, the newly arrived packet preempts the packet under service, i.e., the older packet is discarded.

Our main problem is analytical derivation of average PAoI for the successfully received packets pertinent to a typical node. To this end, we only consider average PAoI of the first node (node 1), thus we only concentrate on the packets of the first node that are received successfully at the destination. We call such packets in the network as tagged packets. All formulas could be derived similarly for the other nodes. Let $T_{i}$ denote the end of the delivery time slot of the $i$-th tagged packet and $H_{i}$ be the time elapsed between receptions of two consecutive tagged packets of node 1. Then, we have $H_{i}=T_{i}-T_{i-1}$. Let $Y_{i}$ and $A_{i}$ be the transmission delay of the $i$-th tagged packet, i.e., the duration between the tagged packet generation and its delivery, and local peak of AoI just before receiving the tagged packet, respectively (see Fig. 3). From Fig. 3, we have $A_{i}=H_{i}+Y_{i-1}$. So, to find the average PAoI, we can write the following equation (due to stationary process for transmission delay of node 1 packets, i.e., $\left.\mathbb{E}\left(Y_{i}\right)=\mathbb{E}\left(Y_{i-1}\right)\right)$ :

$$
\mathbb{E}\left(A_{i}\right)=\mathbb{E}\left(H_{i}\right)+\mathbb{E}\left(Y_{i-1}\right)=\mathbb{E}\left(H_{i}\right)+\mathbb{E}\left(Y_{i}\right)
$$

To find average PAoI in this network, we need to compute each summand on the right hand side in (2). Because of interaction among queues as the result of probable collisions, each node cannot be considered distinctly, so we need to consider the states of all nodes, simultaneously. In the next 


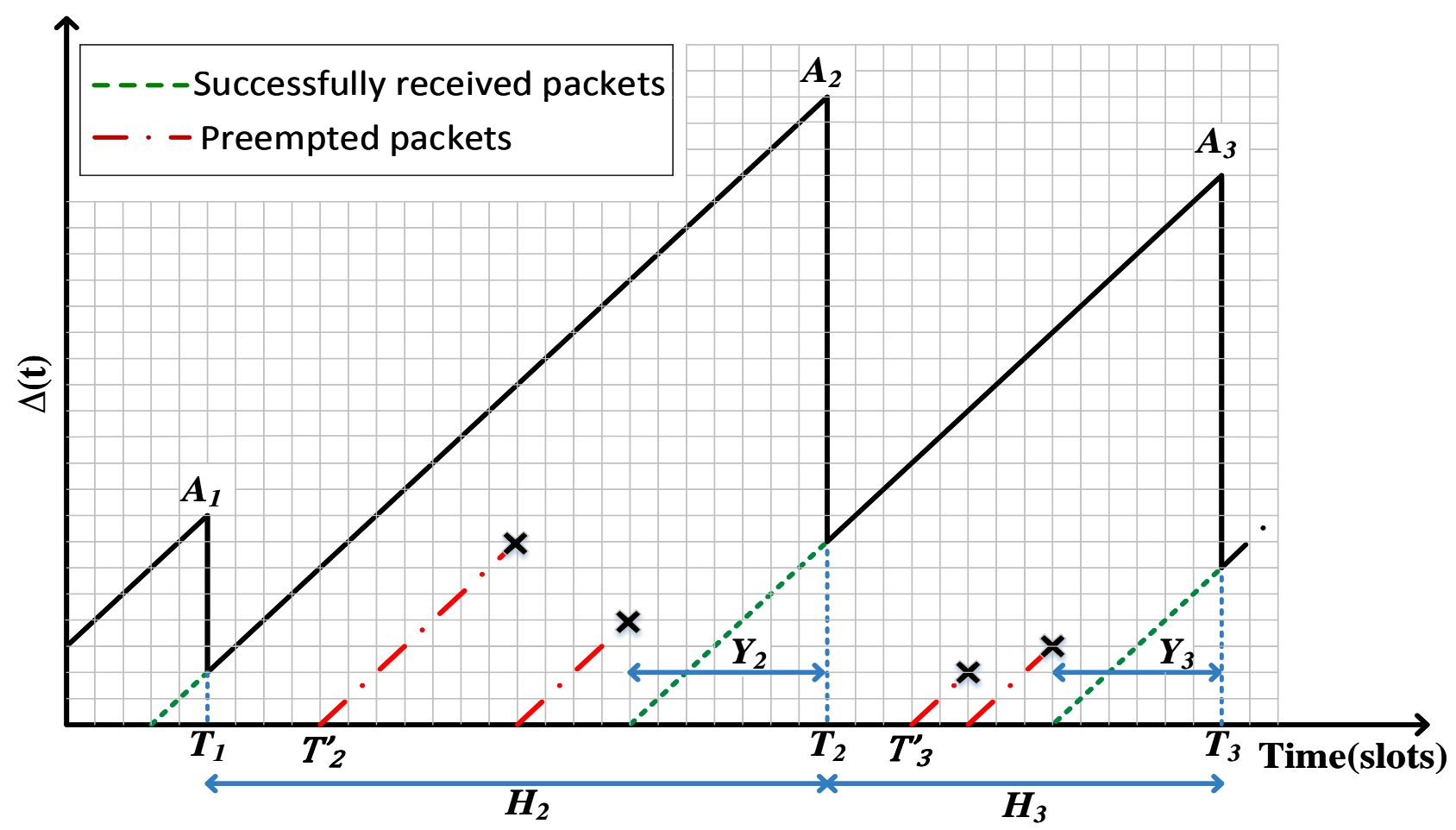

Fig. 3. An example of AoI function in preemptive policy.

section, we derive the summands in (2) in our scenario for the case that $M=2$, and later we will evaluate average PAoI in the network comprised of an arbitrary number of nodes.

\section{PAoI In A Two -Node SLOTTED ALOHA NETwork}

In this section, we find average PAoI in our slotted ALOHA network with two nodes. To this end, we first find $\mathbb{E}\left(H_{i}\right)$ in (2). As mentioned in the previous section, we only concentrate on node 1's PAoI, since all the formulas could be derived similarly for node 2 . Before computing $\mathbb{E}\left(H_{i}\right)$, we first model our network with a discrete time Markov chain (DTMC). As explained in the previous section, each node is considered to just have a server and no buffer. Thus, each node could have one of the states of being empty (does not have a packet at its server) or being full (its server is occupied by a packet) at the beginning of a time slot. Note that for considering the probable collisions, knowing these states is sufficient. By knowing the current state of the network, i.e., knowing that each node is empty or full, the future of our slotted ALOHA network is independent from its past. So, it could be modeled as a DTMC with four states as in Fig. 4. We 


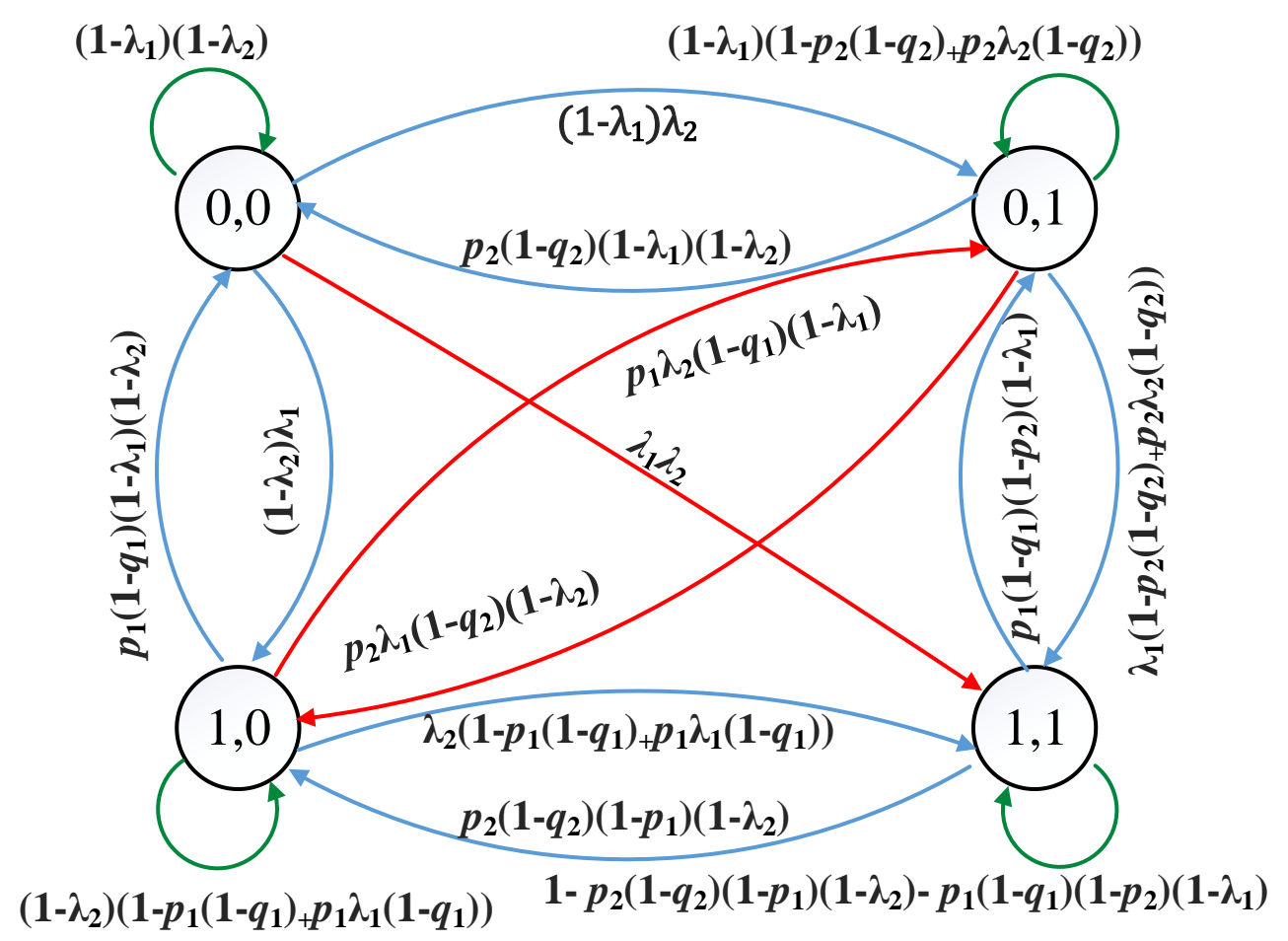

Fig. 4. DTMC of slotted ALOHA network with two nodes.

Show the empty state with 0 and full state with 1 , in the remaining of the paper. For example, $(1,0)$ shows the state where node 1 is full and node 2 is empty. It is important to note that packet preemption does not affect the state of the network, as only emptiness and fullness of nodes is important in our DTMC modeling. The steady state probability of state $(i, j)$, i.e., $\pi_{(i, j)}$, could be found by solving the global balance equations (GBE) of this DTMC.

Let $d_{\bar{S}}(\bar{S} \in\{0,1\})$ be the probability of a successful transmission for node 1 in a time slot in which at its beginning node 1 is full and the state of node 2 has been equal to $\bar{S}$. We have

$$
\begin{gathered}
d_{0}=p_{1}\left(1-q_{1}\right), \\
d_{1}=p_{1}\left(1-q_{1}\right)\left(1-p_{2}\right),
\end{gathered}
$$

since node 1 transmits its packet successfully, i.e., without erasure, with probability $p_{1}\left(1-q_{1}\right)$ if node 2 is empty, otherwise, we also need no collision (i.e., no transmission of node 2 that occurs with probability $\left.\left(1-p_{2}\right)\right)$, in order to have a successful transmission for node 1 .

We are now able to find the probability of a successful transmission of node 1 at a typical time slot as 


$$
P_{\text {success }}=\pi_{(1,0)} d_{0}+\pi_{(1,1)} d_{1}=\left[\begin{array}{l}
d_{0} \\
d_{1}
\end{array}\right]^{\mathrm{T}}\left[\begin{array}{l}
\pi_{(1,0)} \\
\pi_{(1,1)}
\end{array}\right]=\mathbf{d}^{\mathrm{T}} \boldsymbol{\pi}_{(\mathbf{1})}
$$

where $\mathbf{d} \triangleq\left[\begin{array}{l}d_{0} \\ d_{1}\end{array}\right]$ and $\boldsymbol{\pi}_{(\mathbf{1})} \triangleq\left[\begin{array}{l}\pi_{(1,0)} \\ \pi_{(1,1)}\end{array}\right]$. Actually, for having a successful transmission at node 1 at a typical time slot, the state of the nodes should be either $(1,0)$ or $(1,1)$ at the beginning of this slot, as node 1 should have a packet to transmit. On the other hand, $P_{\text {success }}$ is the throughput of node 1 , since the throughput is equal to the number of transmitted packets over a long time interval. Thus, the ergodicity of the DTMC results in the equality of $P_{\text {success }}$ and the throughput of node 1 . $\mathbb{E}\left(H_{i}\right)$ is the expected time elapsed between two consecutive transmissions of node 1 , which is equal to inverse of the throughput of node 1 . Thus, we have

$$
\mathbb{E}\left(H_{i}\right)=\frac{1}{P_{\text {success }}}=\frac{1}{\mathbf{d}^{\mathrm{T}} \boldsymbol{\pi}_{(1)}} .
$$

Now, we find $\mathbb{E}\left(Y_{i}\right)$ as another effective term in (2). This could be challenging due to interactions among the nodes as well as packet preemption. Consider that $T_{i}{ }^{\prime}$ shows the beginning of the first time slot within interval $H_{i}$ that node 1 is not empty. As shown in Fig. 3, some packets of node 1 may be preempted during interval $H_{i}$, according to preemption policy. Moreover, state of node 2 may change during the interval $\left[T_{i}{ }^{\prime}, T_{i}\right]$. Thus, the probability of successful transmission of node 1 would change between $d_{0}$ and $d_{1}$ during this interval, as state of node 2 may change.

Let $t_{\overline{S^{\prime}}, \bar{S}}\left(\bar{S}, \bar{S}^{\prime} \in\{0,1\}\right)$ be the probability that at a time slot which node 1 is full at its beginning, node 1 packet does not leave the system (i.e., not being successfully transmitted or preempted) and the state of node 2 transits from $\overline{S^{\prime}}$ to $\bar{S}$ during this time slot. This would happen if and only if all of the following three events happen together:

1- Node 1 does not transmit its packet successfully during this time slot, due to collision, randomized access of node 1 to the channel (i.e., not transmitting in that time slot), or channel erasure.

2- The current packet of node 1 is not preempted at the end of this time slot. Thus, no new packet has been arrived at node 1 during this time slot. 
3- The state of node 2 transits from $\overline{S^{\prime}}$ to $\bar{S}$ during this time slot.

For example, $t_{0,0}$ is the probability that at a time slot which node 1 is full at its beginning, node 1 packet does not leave the system, while node 2 is empty at the beginning and end of this time slot (i.e., at two consecutive slot boundaries). To find this probability, we need to find the probabilities of the aforementioned three events. The probability that node 1 does not update its status (i.e., does not transmit its packet successfully) is $\left(1-p_{1}\left(1-q_{1}\right)\right)$, since node 2 is empty at the beginning of this time slot. The packet of node 1 is not preempted with probability $\left(1-\lambda_{1}\right)$ and node 2 is still empty at the end of this time slot with probability $\left(1-\lambda_{2}\right)$. Hence, we have

$$
t_{0,0}=\left(1-\lambda_{1}\right)\left(1-p_{1}\left(1-q_{1}\right)\right)\left(1-\lambda_{2}\right)
$$

We also have

$$
\begin{gathered}
t_{1,0}=\left(1-\lambda_{1}\right)\left(1-\lambda_{2}\right) p_{2}\left(1-q_{2}\right)\left(1-p_{1}\right), \\
t_{0,1}=\left(1-\lambda_{1}\right) \lambda_{2}\left(1-p_{1}\left(1-q_{1}\right)\right) \\
t_{1,1}=\left(1-\lambda_{1}\right)\left[p_{1} p_{2}+p_{1} q_{1}\left(1-p_{2}\right)+\left(1-p_{2}\left(1-q_{2}\right)\left(1-\lambda_{2}\right)\right)\left(1-p_{1}\right)\right] .
\end{gathered}
$$

For another example, $t_{1,1}$ is the probability that at a time slot which node 1 is full at its beginning, node 1 packet does not leave the system, while node 2 is full at both beginning and end of this time slot. In justifying (10), similar to proof of $(7),\left(1-\lambda_{1}\right)$ is the probability that node 1 packet is not preempted. In addition, when node 1 does not update its status while node 2 is still full at the end of a time slot, either a collision has happened during the slot (its probability equals $p_{1} p_{2}$ ), or only node 1 has sent its packet but it has become erased at destination (with probability $p_{1} q_{1}\left(1-p_{2}\right)$ ) or node 1 has not gotten access to the channel (with probability $\left.\left(1-p_{1}\right)\right)$ and node 2 server is still full at the end of the time slot, which happens with probability $\left(1-p_{2}\left(1-q_{2}\right)\left(1-\lambda_{2}\right)\right)$. Hence, multiplying $\left(1-\lambda_{1}\right)$ and $\left[p_{1} p_{2}+p_{1} q_{1}\left(1-p_{2}\right)+(1-\right.$ $\left.\left.p_{2}\left(1-q_{2}\right)\left(1-\lambda_{2}\right)\right)\left(1-p_{1}\right)\right]$, results in (10). We leave validation of (8) and (9) to the reader, though in the next section we will bring the general proof of these equations when the number of nodes is arbitrary. 
To confront the interaction among the nodes and find $\mathbb{E}\left(Y_{i}\right)$, let $G_{n, \bar{S}}(\bar{S} \in\{0,1\})$ be the probability that the system time (the time that a packet is in the system until being successfully transmitted or being preempted) of a typical node 1 packet exceeds $n$ slots and at the beginning of the next slot (i.e., the $(n+1)$-th slot after arrival of this packet), the state of node 2 be equal to $\bar{S}$. Our main intention is to compute $G_{n, \bar{S}}$ recursively. We have

$$
\begin{aligned}
& G_{n, 0}=t_{0,0} G_{n-1,0}+t_{1,0} G_{n-1,1} \\
& G_{n, 1}=t_{0,1} G_{n-1,0}+t_{1,1} G_{n-1,1}
\end{aligned}
$$

In justifying (11), we divide $G_{n, 0}$ into two probabilities $G_{n-1,0}$ and $G_{n-1,1}$ in which system time of a typical packet exceeds $(n-1)$ slots and state of node 2 at the beginning of the $n$-th slot of system time is empty and full, respectively. The second summand in (11), indicates the probability that during the $n$-th slot of system time, the packet does not leave the system and state of node 2 transits from full to empty state (which happens with probability $t_{1,0}$ ). Thus, multiplication of $t_{1,0}$ and $G_{n-1,1}$ comprise the second summand of (11). The first summand could be validated by considering the interpretation of $G_{n-1,0}$. With the same manner, one could prove (12). Consider that

$$
g_{n}=G_{n, 0}+G_{n, 1}
$$

where $g_{n}$ denotes the probability that the system time of a typical packet at node 1 exceeds $n$ slots. Hence, $g_{n}$ is the complement cumulative distribution function (CCDF) of system time of node 1 packets. It could be useful to have a graphical view over (11)-(12). In Fig. 5, we use a trellis-based modeling approach to show these equations graphically. This figure shows that $G_{n, 0}$ is equal to sum of multiplication of $G_{n-1,0}$ and $G_{n-1,1}$ with the corresponding factors. Actually, this figure shows that how we divide the CCDF of system time of node 1 packets, i.e., $g_{n}$, into two sequences of $G_{n, 0}$ and $G_{n, 1}$ to handle the interaction among the nodes.

Eqns. (11) and (12) could be written in matrix form as

$$
\mathbf{G}_{n}=\left[\begin{array}{l}
G_{n, 0} \\
G_{n, 1}
\end{array}\right]=\left[\begin{array}{ll}
t_{0,0} & t_{1,0} \\
t_{0,1} & t_{1,1}
\end{array}\right]\left[\begin{array}{c}
G_{n-1,0} \\
G_{n-1,1}
\end{array}\right]=\mathbf{t G}_{n-1}
$$




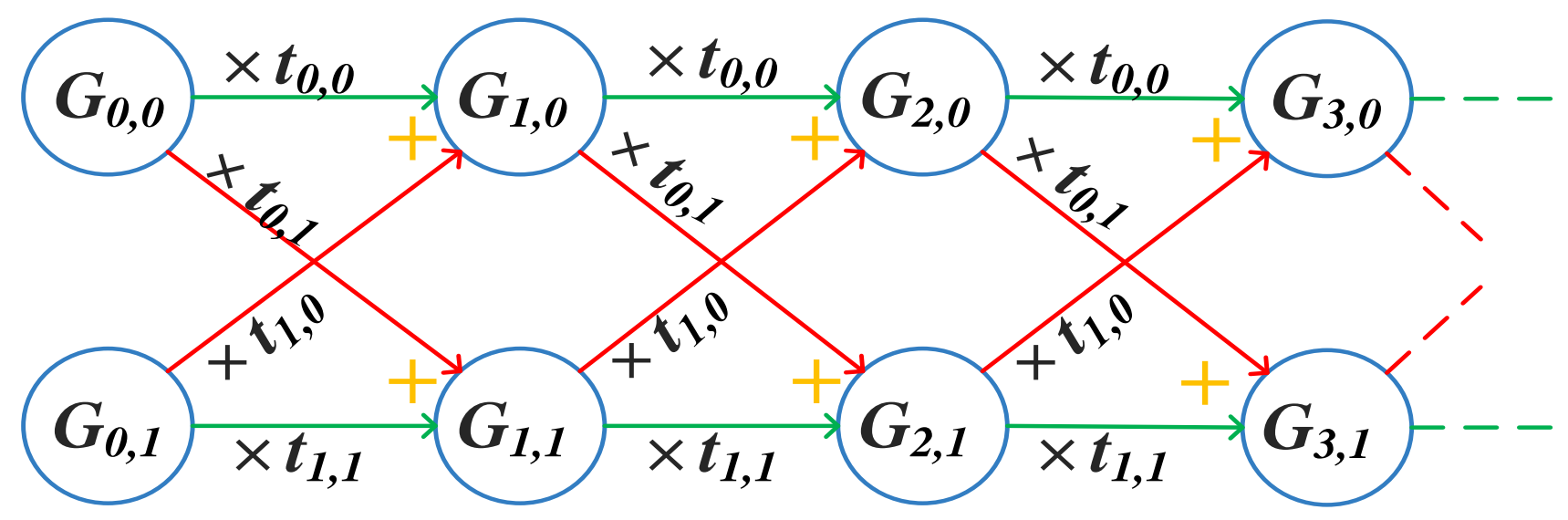

Fig. 5. Trellis form of (11) and (12).

where $\mathbf{t} \triangleq\left[\begin{array}{ll}t_{0,0} & t_{1,0} \\ t_{0,1} & t_{1,1}\end{array}\right]$. Thus, we have

$$
\mathbf{G}_{n}=\mathbf{t}^{n} \mathbf{G}_{0}
$$

Hence, it is enough to find $\mathbf{G}_{0}$ in order to obtain $\mathbf{G}_{n}$ for $n>0$. We need to find the initial values of $G_{0,0}$ and $G_{0,1}$ to find $\mathbf{G}_{0}$. Note that $G_{0,0}$ is the probability that in the beginning of the first time slot after arrival of a typical packet at node 1 , state of node 2 is empty. As packets arrive at node 1 according to a Bernoulli process, hence from BASTA theorem (Bernoulli arrivals see time averages, for more details see [31]) $G_{0,0}$ is the steady state probability of emptiness of node 2 . Thus, we have

$$
G_{0,0}=\pi_{(0,0)}+\pi_{(1,0)}
$$

Similarly, we have

$$
G_{0,1}=\pi_{(0,1)}+\pi_{(1,1)}
$$

Then, we have

$$
\mathbf{G}_{0}=\left[\begin{array}{l}
G_{0,0} \\
G_{0,1}
\end{array}\right]=\left[\begin{array}{l}
\pi_{(0,0)}+\pi_{(1,0)} \\
\pi_{(0,1)}+\pi_{(1,1)}
\end{array}\right]
$$

We are now able to evaluate $\mathbb{E}\left(Y_{i}\right)$. Let $e_{n}$ be the probability that a typical packet being transmitted successfully at the $n$-th slot after its arrival. So, its system time equals $n$. Then, we have 


$$
e_{n}=d_{0} G_{n-1,0}+d_{1} G_{n-1,1}=\mathbf{d}^{\mathrm{T}} \mathbf{G}_{n-1}=\mathbf{d}^{\mathrm{T}} \mathbf{t}^{n-1} \mathbf{G}_{0} .
$$

This could be proved by conditioning on the state of node 2 at the $n$-th slot of the packet service time. With probability $G_{n-1,0}$, a typical packet has not been transmitted or been preempted in the last $(n-1)$ slots of the system time, while in the next slot, node 2 is empty. In this case, node 1 could successfully transmit its packet in the next slot with probability $d_{0}$. Hence, multiplication of $G_{n-1,0}$ and $d_{0}$ comprises the first summand in (19). Similarly, the second summand in (19) could be investigated by considering the case that node 2 is full at the beginning of the $n$-th slot of the system time of node 1 packet. In the second equality of (19), we rewrote the previous part in matrix form and in the last equality, we used (15) and replaced $\mathbf{G}_{n-1}$ by $\mathbf{t}^{n-1} \mathbf{G}_{0}$.

Note that $Y_{i}$ is the transmission time of the $i$-th tagged packet. Thus, $P\left(Y_{i}=n\right)$ is the probability that the system time of a node 1 packet equals $n$, provided that this packet has not been preempted by any other packet. Then according to Bayes' rule, we have

$$
P\left(Y_{i}=n\right)=\frac{e_{n}}{\sum_{n=0}^{\infty} e_{n}} .
$$

Hence, by replacing $e_{n}$ from (20), we have

$$
\begin{aligned}
\mathbb{E}\left(Y_{i}\right)=\frac{\sum_{n=1}^{\infty} n \mathbf{d}^{\mathrm{T}} \mathbf{t}^{n-1} \mathbf{G}_{0}}{\sum_{n=1}^{\infty} \mathbf{d}^{\mathrm{T}} \mathbf{t}^{n-1} \mathbf{G}_{0}}=\frac{\mathbf{d}^{\mathrm{T}}\left(\sum_{n=1}^{\infty} n \mathbf{t}^{n-1}\right) \mathbf{G}_{0}}{\mathbf{d}^{\mathrm{T}}\left(\sum_{n=1}^{\infty} \mathbf{t}^{n-1}\right) \mathbf{G}_{0}} & =\frac{\mathbf{d}^{\mathrm{T}}\left(\sum_{n=1}^{\infty} \mathbf{t}^{n-1}\right)\left(\sum_{n=1}^{\infty} \mathbf{t}^{n-1}\right) \mathbf{G}_{0}}{\mathbf{d}^{\mathrm{T}}\left(\sum_{n=1}^{\infty} \mathbf{t}^{n-1}\right) \mathbf{G}_{0}} \\
& =\frac{\mathbf{d}^{\mathrm{T}}\left(\mathbf{I}_{2 \times 2}-\mathbf{t}\right)^{-1}\left(\mathbf{I}_{2 \times 2}-\mathbf{t}\right)^{-1} \mathbf{G}_{0}}{\mathbf{d}^{\mathrm{T}}\left(\mathbf{I}_{2 \times 2}-\mathbf{t}\right)^{-1} \mathbf{G}_{0}}
\end{aligned}
$$

where $\mathbf{I}_{2 \times 2}$ is the identity matrix with dimension of two. It is important to note that since the PAoI in investigating network is finite, therefore $\sum_{n=1}^{\infty} \mathbf{t}^{n-1}$ would not be divergent and could be replaced by $\left(\mathbf{I}_{2 \times 2}-\mathbf{t}\right)^{-1}$. Hence, from (2), (6) and (21), average PAoI could be derived as

$$
\mathbb{E}\left(A_{i}\right)=\frac{1}{\mathbf{d}^{\mathrm{T}} \boldsymbol{\pi}_{(1)}}+\frac{\mathbf{d}^{\mathrm{T}}\left(\mathbf{I}_{2 \times 2}-\mathbf{t}\right)^{-1}\left(\mathbf{I}_{2 \times 2}-\mathbf{t}\right)^{-1} \mathbf{G}_{0}}{\mathbf{d}^{\mathrm{T}}\left(\mathbf{I}_{2 \times 2}-\mathbf{t}\right)^{-1} \mathbf{G}_{0}} .
$$

In the next section, we will generalize the analysis of this section to a slotted ALOHA network with $M$ buffer-less nodes. 


\section{PAoI in Slotted ALOHA NeTwork With $M$ NodeS}

In this section, we evaluate average PAoI in a slotted ALOHA network with $M$ buffer-less nodes. We follow the same approach as in the previous section. We first model our network with a DTMC similar to one for the case $M=2$. As explained in the previous section, each node just have a single server. Thus, each node could have one of the states of being empty (does not have a packet at its server) or being full (the server is occupied by a packet) in the beginning of a time slot. So, this network could be modeled as a DTMC with $2^{M}$ states. Let $S \in\{0,1\}^{M}$ be a vector of $M$ binary elements like $\left(S_{1}, S_{2}, \ldots, S_{M}\right)$, where $S_{i}$ shows the current state of node $i .0$ denotes the emptiness of the node and 1 shows its fullness. Let $f_{a}(x)$ determine the binary representation of decimal number $x$ with $a$ digits. For example, $f_{3}(0)=(0,0,0)$, or $f_{3}(7)=(1,1,1)$. This notation helps to present state of the nodes. For example, $S=f_{M}(0)$ shows the state where all nodes are empty and $S=f_{M}\left(2^{M}-1\right)$ determines the state where all nodes are full.

Similar to previous section, for analyzing PAoI at node 1, it is necessary to consider the state of the other nodes. Thus, let $\bar{S} \in\{0,1\}^{M-1}$ be the vector that shows the state of all nodes except node 1. Actually, $\bar{S}=\left(S_{2}, S_{3}, \ldots, S_{M}\right)$ or $S=\left(S_{1}, \bar{S}\right)$. For example, $\bar{S}=f_{M-1}(0)$ determines that nodes $2,3, \ldots, M$ are empty. We consider $S^{\prime}=\left(S_{1}{ }^{\prime}, \overline{S^{\prime}}\right)$ as an arbitrary vector of states of all nodes which does not have any specific relation with $S$. To find the transition probabilities of the DTMC, let $A_{\left(S, S^{\prime}\right)}$ be a set defined over vectors $S$ and $S^{\prime}$, as

$$
A_{\left(S, S^{\prime}\right)}=\left\{i \mid 1 \leq i \leq M, S_{i}=0, S_{i}^{\prime}=1\right\}
$$

Similarly, we define $B_{\left(S, S^{\prime}\right)}$ as

$$
B_{(S, S \prime)}=\left\{i \mid 1 \leq i \leq M, S_{i}=1, S_{i}^{\prime}=0\right\}
$$

and $C_{(S)}$ as

$$
C_{(S)}=\left\{i \mid 1 \leq i \leq M, S_{i}=1\right\}
$$

Let $p_{S, S^{\prime}}$ denote the transition probability from state $S$ to state $S^{\prime}$ in this DTMC. Since in a slotted ALOHA network, at a time slot at most one source could send its packet without collision, 
so in order to have a non-zero $p_{S, S^{\prime}}$, it is required that $\left|B_{\left(S, S^{\prime}\right)}\right|=0$ or $\left|B_{\left(S, S^{\prime}\right)}\right|=1$, otherwise we have $p_{S, S^{\prime}}=0$. For the case $\left|B_{\left(S, S^{\prime}\right)}\right|=1$, let $\eta$ be the only member of $B_{\left(S, S^{\prime}\right)}$. Thus,

$$
p_{S, S^{\prime}}=p_{\eta}\left(1-q_{\eta}\right)\left(1-\lambda_{\eta}\right) \prod_{i \in C_{(S), i \neq \eta}}\left(1-p_{i}\right) \prod_{j \in A} \lambda_{\left(S, S^{\prime}\right)} \prod_{l \notin A_{(S, S \prime)} \cup C_{(S)}}\left(1-\lambda_{l}\right)
$$

When $\left|B_{\left(S, S^{\prime}\right)}\right|=0$, all of the nodes that are currently full, should still have a packet in the beginning of the next time slot. Thus, we have

$$
p_{S, S^{\prime}}=\left[1-\sum_{k \in C_{(S)}}\left(p_{k}\left(1-q_{k}\right)\left(1-\lambda_{k}\right) \prod_{i \in C_{(S)}, i \neq k}\left(1-p_{i}\right)\right)\right] \prod_{j \in A_{(S, S \prime)}} \lambda_{j} \prod_{l \notin A_{(S, S \prime} \cup C_{(S)}}\left(1-\lambda_{l}\right) .
$$

Since the number of states is finite, the steady state probability of being in state $S$ at the beginning of a time slot, i.e., $\pi(S)$, could be found by solving the global balance equations (GBE).

Let $d_{\bar{S}}$ be the probability of a successful transmission for node 1 in a time slot that node 1 is full and state of the other nodes is equal to $\bar{S}$. We have

$$
d_{\bar{S}}=p_{1}\left(1-q_{1}\right) \prod_{i \in C}\left(1-p_{i}\right)
$$

As node 1 should transmit its packet with probability $p_{1}$, the other full nodes should not transmit their packets (happening with probability $\prod_{i \in C_{((1, \bar{S}))}, i \neq 1}\left(1-p_{i}\right)$ ) and the transmitted packet of node 1 should be decoded at destination (happening with probability $\left(1-q_{1}\right)$ ). Similar to previous section, let $\mathbf{d}$ be the vector comprised of $d_{\bar{S}}\left(\bar{S} \in\{0,1\}^{M-1}\right)$, as shown in the following:

$$
\mathbf{d} \triangleq\left[\begin{array}{c}
d_{f_{M-1}(0)} \\
d_{f_{M-1}(1)} \\
\vdots \\
d_{f_{M-1}\left(2^{M-1}-1\right)}
\end{array}\right]
$$

Similar to previous section, we define vector $\boldsymbol{\pi}_{(\mathbf{1})}$ as

$$
\boldsymbol{\pi}_{(1)} \triangleq\left[\begin{array}{c}
\pi_{\left(1, f_{M-1}(0)\right)} \\
\pi_{\left(1, f_{M-1}(1)\right)} \\
\vdots \\
\pi_{\left(1, f_{M-1}\left(2^{M-1}-1\right)\right)}
\end{array}\right]
$$

Hence, the probability of a successful transmission of node 1 at a typical time slot is $\mathbf{d}^{\mathrm{T}} \mathbf{\pi}_{(1)}$, since 
with probability $\pi_{(1, \bar{S})}$, the state of nodes is equal to $(1, \bar{S})$ and subject to this situation, node 1 could deliver its packet successfully during this time slot with probability $d_{\bar{S}}$. With the same argument as in the previous section, $\mathbb{E}\left(H_{i}\right)$ could be computed from (6).

Let $t_{\overline{S^{\prime}}, \bar{S}}\left(\bar{S}, \overline{S^{\prime}} \in\{0,1\}^{M-1}\right)$ be the probability that at a time slot which at its beginning node 1 is full, packet of node 1 does not leave the system (i.e., neither be transmitted successfully nor be preempted) and state of the other nodes transits from $\bar{S}^{\prime}$ to $\bar{S}$ during this time slot. Hence, we have

$$
t_{\overline{S^{\prime}, \bar{S}}}=\left(1-\lambda_{1}\right)\left[p_{\left(1, \overline{S^{\prime}}\right),(1, \bar{S})}-\frac{\lambda_{1} p_{\left(1, \overline{S^{\prime}}\right),(0, \bar{S})}}{1-\lambda_{1}}\right]
$$

In fact, similar to previous section, the packet of node 1 is not preempted with probability $\left(1-\lambda_{1}\right)$. In addition, state of all nodes should transit from $\left(1, \overline{S^{\prime}}\right)$ to $(1, \bar{S})$ while node 1 cannot transit its packet successfully. In some transition from state $\left(1, \bar{S}^{\prime}\right)$ to $(1, \bar{S})$, it is probable that node 1 successfully transmits its packet and then a new packet arrives at node 1 at the same slot (note to the ED-LA convention). The probability of such a transition is $\left(\lambda_{1} p_{\left(1, \overline{S^{\prime}}\right),(0, \bar{S})}\right) /\left(1-\lambda_{1}\right)$, because this probability is equal to the probability of transition from state $\left(1, \overline{S^{\prime}}\right)$ to $(0, \bar{S})$ such that instead of not having an arrival at node 1 at this slot (with probability $1-\lambda_{1}$ ), we have an arrival (with probability $\left.\lambda_{1}\right)$. Hence, $\left[p_{\left(1, \overline{S^{\prime}}\right),(1, \bar{S})}-\left(\lambda_{1} p_{\left(1, \overline{S^{\prime}}\right),(0, \bar{S})}\right) /\left(1-\lambda_{1}\right)\right]$ is the probability of transition from state $\left(1, \overline{S^{\prime}}\right)$ to $(1, \bar{S})$, while node 1 does not transmit successfully. Thus, multiplication of $\left(1-\lambda_{1}\right)$ and $\left[p_{\left(1, \overline{S^{\prime}}\right),(1, \bar{S})}-\left(\lambda_{1} p_{\left(1, \overline{S^{\prime}}\right),(0, \bar{S})}\right) /\left(1-\lambda_{1}\right)\right]$ is the probability that at the typical time slot, node 1 packet does not leave the system while state of the other nodes transits from $\overline{S^{\prime}}$ to $\bar{S}$ during the slot, which proves (31). Similar to previous section, we define matrix t, as

$$
\mathbf{t} \triangleq\left[\begin{array}{ccc}
t_{f_{M-1}(0), f_{M-1}(0)} & \cdots & t_{f_{M-1}\left(2^{M-1}-1\right), f_{M-1}(0)} \\
\vdots & \ddots & \vdots \\
t_{f_{M-1}(0), f_{M-1}\left(2^{M-1}-1\right)} & \cdots & t_{f_{M-1}\left(2^{M-1}-1\right), f_{M-1}\left(2^{M-1}-1\right)}
\end{array}\right]
$$

Now, we first find the CCDF of system time for a typical packet of node 1 . Let $G_{n, \bar{S}}$ be the probability that system time of the typical packet of node 1 exceeds $n$ slots and the beginning of the $(n+1)$-th slot of its system time, the state of nodes $2,3, \ldots, M$ be equal to $\bar{S}$. We have 


$$
G_{n, \bar{S}}=\sum_{\overline{S^{\prime} \in\{0,1\}^{M-1}}} G_{n-1, \overline{S^{\prime}}} t_{\overline{S^{\prime}, \bar{S}}}
$$

For the proof, let $\varepsilon_{n, \bar{S}}$ be the event that system time of a typical packet exceeds $n$ slots while at the beginning of the next slot, the state of nodes $2,3, \ldots, M$ is equal to $\bar{S}$. The probability of $\varepsilon_{n, \bar{S}}$ subject to event $\varepsilon_{n-1, \bar{S} \prime}$ is $t_{\overline{S^{\prime},}, \bar{S}}$, since in this case, the packet of node 1 does not leave the system during the $n$-th slot of its system time and state of the other nodes transits from $\overline{S^{\prime}}$ to $\bar{S}$. As $G_{n-1, \overline{S^{\prime}}}$ is the probability of event $\varepsilon_{n-1, \overline{S^{\prime}}}$, (33) is justified according to the total probability theorem. By defining $\mathbf{G}_{n}$ as

$$
\mathbf{G}_{n} \triangleq\left[\begin{array}{c}
G_{n, f_{M-1}(0)} \\
G_{n, f_{M-1}(1)} \\
\vdots \\
G_{n, f_{M-1}\left(2^{M-1}-1\right)}
\end{array}\right]
$$

from (33) we have

$$
\mathbf{G}_{n}=\mathbf{t G}_{n-1}=\mathbf{t}^{n-1} \mathbf{G}_{0}
$$

Similar to the previous section, we have

$$
\mathbf{G}_{0}=\left[\begin{array}{c}
\pi_{\left(0, f_{M-1}(0)\right)}+\pi_{\left(1, f_{M-1}(0)\right)} \\
\pi_{\left(0, f_{M-1}(1)\right)}+\pi_{\left(1, f_{M-1}(1)\right)} \\
\vdots \\
\pi_{\left(0, f_{M-1}\left(2^{M-1}-1\right)\right)}+\pi_{\left(1, f_{M-1}\left(2^{M-1}-1\right)\right)}
\end{array}\right] .
$$

Actually, $G_{0, \bar{S}}$ is the probability that at the beginning of the first time slot after arrival of a typical packet at node 1 , state of the other nodes is equal to $\bar{S}$. As the arrival process at node 1 is a Bernoulli process, according to BASTA theorem, $G_{0, \bar{S}}$ is equal to steady state probability that the state of nodes $2,3, \ldots, M$ be equal to $\bar{S}$. In other words, $G_{0, \bar{S}}=\pi_{(0, \bar{S})}+\pi_{(1, \bar{S})}$.

Similar to (19) in the previous section, let $e_{n}$ be the probability that a typical packet is transmitted successfully at the $n$-th slot after its arrival. We have

$$
e_{n}=\sum_{\bar{S} \in\{0,1\}^{M-1}} G_{n-1, \bar{S}} d_{\bar{S}}=\mathbf{d}^{\mathrm{T}} \mathbf{G}_{n-1}=\mathbf{d}^{\mathrm{T}} \mathbf{t}^{n-1} \mathbf{G}_{0} .
$$

This could be proved by conditioning on the state of nodes $2,3, \ldots, M$ at the $n$-th time slot of the system time of the typical packet at node 1 . With probability $G_{n-1, \bar{S}}$, this packet has not left the 
system in the last $(n-1)$ time slots while the state of nodes $2,3, \ldots, M$ at the next slot equals $\bar{S}$. In this case, the probability that this packet has been successfully transmitted in the $n$-th time slot is equal to $d_{\bar{S}}$. With the same explanation for (20), we have

$$
\begin{aligned}
& \mathbb{E}\left(Y_{i}\right)=\frac{\sum_{n=1}^{\infty} n e_{n}}{\sum_{n=1}^{\infty} e_{n}}=\frac{\mathbf{d}^{\mathrm{T}}\left(\sum_{n=1}^{\infty} n \mathbf{t}^{n-1}\right) \mathbf{G}_{0}}{\mathbf{d}^{\mathrm{T}}\left(\sum_{n=1}^{\infty} \mathbf{t}^{n-1}\right) \mathbf{G}_{0}}=\frac{\mathbf{d}^{\mathrm{T}}\left(\sum_{n=1}^{\infty} \mathbf{t}^{n-1}\right)\left(\sum_{n=1}^{\infty} \mathbf{t}^{n-1}\right) \mathbf{G}_{0}}{\mathbf{d}^{\mathrm{T}}\left(\sum_{n=1}^{\infty} \mathbf{t}^{n-1}\right) \mathbf{G}_{0}}
\end{aligned}
$$

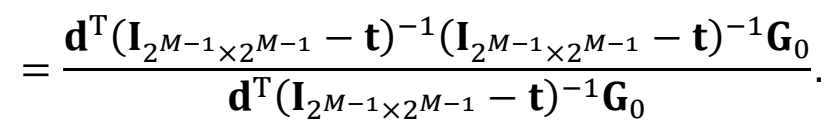

Hence, from (2), (6), and (38), the average PAoI could be derived as

$$
\mathbb{E}\left(A_{i}\right)=\frac{1}{\mathbf{d}^{\mathrm{T}} \boldsymbol{\pi}_{(1)}}+\frac{\mathbf{d}^{\mathrm{T}}\left(\mathbf{I}_{2^{M-1} \times 2^{M-1}}-\mathbf{t}\right)^{-1}\left(\mathbf{I}_{2^{M-1} \times 2^{M-1}}-\mathbf{t}\right)^{-1} \mathbf{G}_{0}}{\mathbf{d}^{\mathrm{T}}\left(\mathbf{I}_{2^{M-1} \times 2^{M-1}}-\mathbf{t}\right)^{-1} \mathbf{G}_{0}} .
$$

It is worth to note that by our trellis-based modeling approach, we could evaluate PAoI in similar networks which could be modeled with a finite state DTMC. In our work, the complexity of computing the average PAoI grows exponentially with the number of nodes, since the number of states in the modeled DTMC is $2^{M}$. In the next section, we will bring some numerical results and validate our analysis.

\section{NumericAl RESUlts}

In this section, we provide some numerical results, based on the analysis in the previous sections. At first, in a symmetric ALOHA network, where the arrival rate (AR), transmission rate (TR) and packet erasure probability of all nodes are represented by $(\lambda, p, q)$, respectively, we compare analytically evaluated PAoI with the simulation results in Fig. 6 . We consider $(\lambda, p, q)=$ $\left(\frac{0.5}{M}, 0.5,0.1\right)$ and sketch the average PAoI versus the number of nodes. We do network simulation over one million time slots. These results confirm our analytic evaluation of the average PAoI.

Next, we concentrate on the slotted ALOHA network comprised of two nodes. For the sake of simplicity, we consider that the erasure probability for both nodes is equal to $0.1\left(q_{1}=q_{2}=0.1\right)$. Let $\Delta_{1}$ and $\Delta_{2}$ denote the average PAoI of nodes 1 and 2 , respectively. In such a network, it is interesting to find the parameter values such that $\bar{\Delta}=\frac{\Delta_{1}+\Delta_{2}}{2}$ does not exceed a specific threshold. 


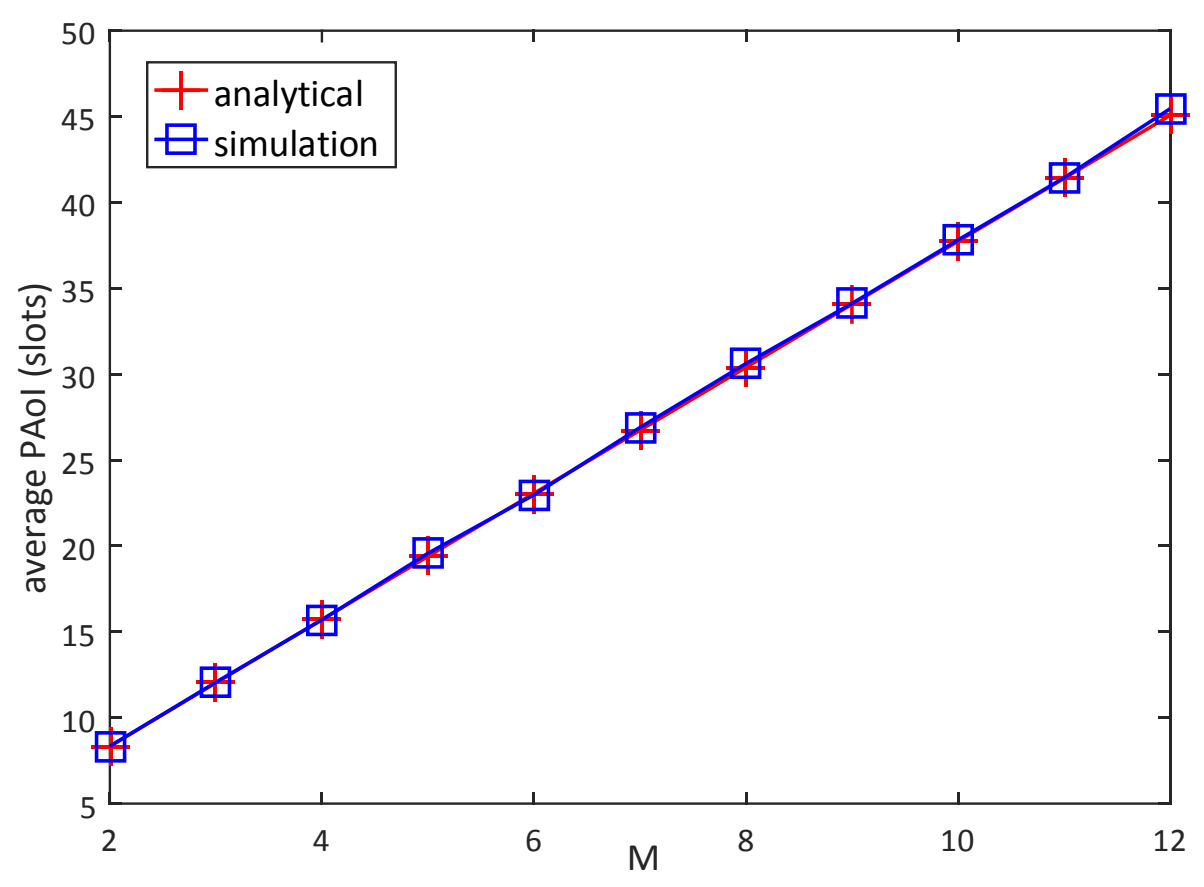

Fig. 6. Comparing the analyticaly evaluated average PAoI of a symmetric slotted ALOHA network $((\lambda, p, q)=$ $\left.\left(\frac{0.5}{M}, 0.5,0.1\right)\right)$ with simulation results.

Since the ARs, i.e., $\lambda_{1}$ and $\lambda_{2}$, and TRs, i.e., $p_{1}$ and $p_{2}$, of both nodes affect their PAoIs, we investigate the regions over ARs and TRs where $\bar{\Delta}$ is constrained to some specific thresholds. We call these regions as PAoI-constrained regions.

We have shown $\bar{\Delta}$ contours in Fig. 7 for different set of parameters, using the closed-form relation obtained in Section III. In Figs. 7(a) and 7(b) the contours specify the boundary of the regions over ARs where $\bar{\Delta}$ does not exceed some specific thresholds, respectively. Some of these contours do not seem to be closed, which means $\lambda_{1}=1$ and $\lambda_{2}=1$, i.e., the maximum rates of Bernoulli processes, are the other boundaries of these regions. For more clearance, the $\bar{\Delta}$-limited regions for $\bar{\Delta}=6$ have been hatched in Fig. 7. Similar contours in Figs. 7(c) and 7(d) specify the boundary of the regions over TRs. We observe that these constrained regions are convex. Also, by increasing the threshold, a wider range of ARs and TRs are included in the constrained PAoI regions. Moreover, Fig. 7(b) shows that if TR of node 1 is lower than TR of node 2 , the $\bar{\Delta}$ constrained regions mostly cover the points where AR of node 1 is relatively higher than AR of 


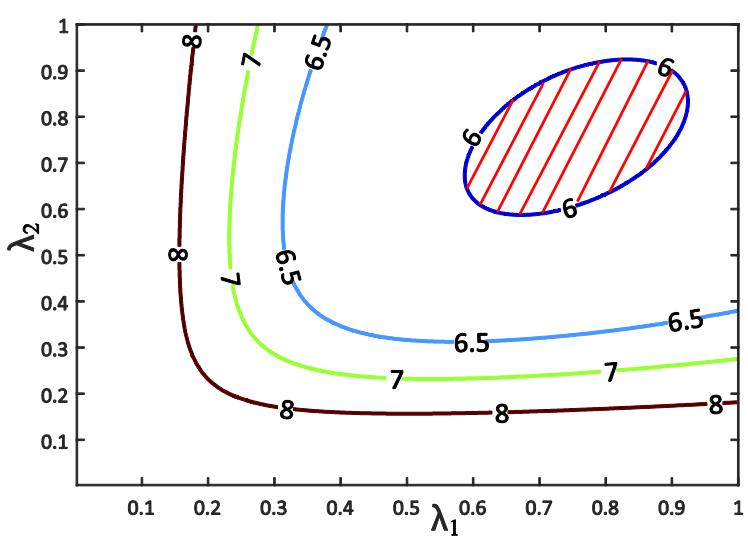

(a) $p_{1}=p_{2}=0.65$

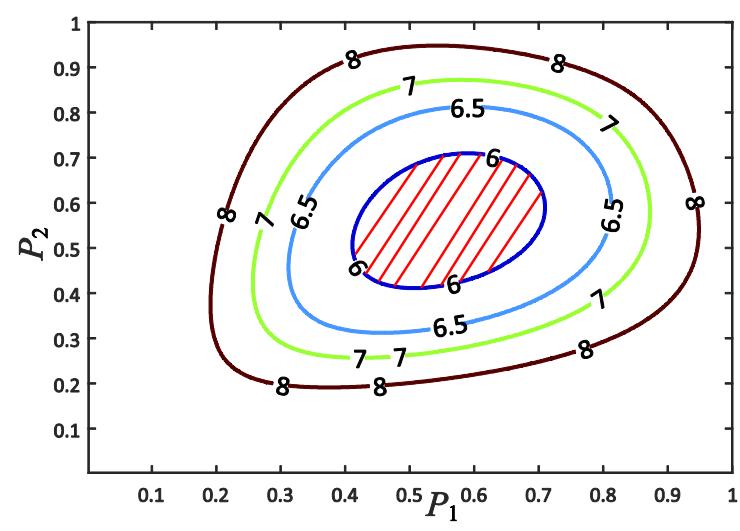

(c) $\lambda_{1}=\lambda_{2}=0.65$

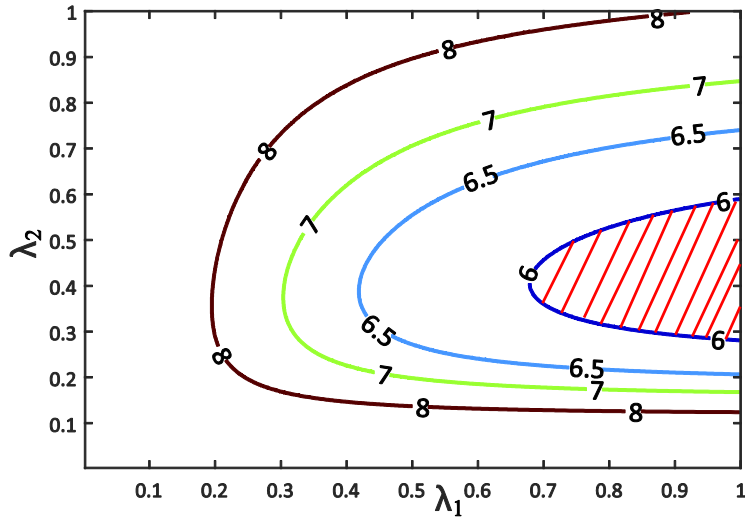

(b) $p_{1}=0.5, p_{2}=0.8$

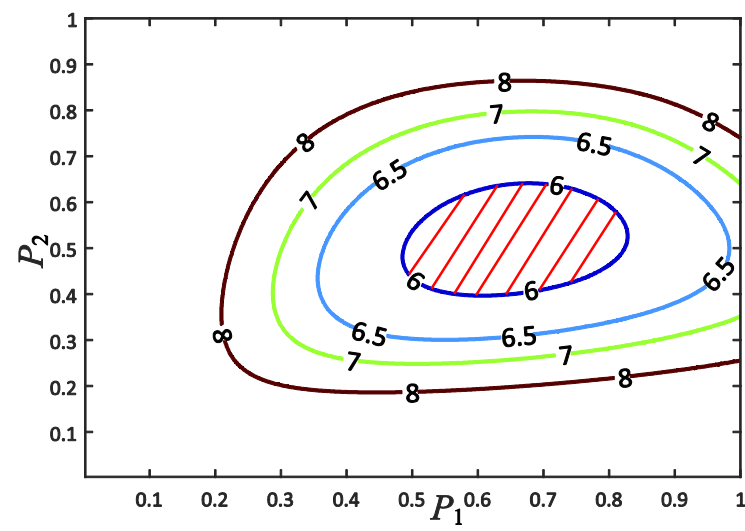

(d) $\lambda_{1}=0.5, \lambda_{2}=0.8$

Fig. 7. $\bar{\Delta}$-constrained regions.

node 2 . This makes a balance between the nodes with different TRs, as average PAoI of both nodes is equally important in $\bar{\Delta}$. The same result could be concluded for $\bar{\Delta}$-constrained regions over TRs, as observed in Fig. 7(d).

In Fig. 8, we have studied average PAoI for a symmetric network for different values of $(\lambda, p)$, varied by the step size 0.01 , in a network with two nodes. The optimal value of $(\lambda, p)$ that minimizes the average PAoI has been shown on the figure. For some other number of nodes, the result of the same study could be observed in Table 1. It is observed that to minimize the average PAoI of a symmetric network, $(1,1 / M)$ is the optimal choice for $(\lambda, p)$. It is important to note that $\lambda=1$, is not the optimal AR for any choice of $p$, as observed in Fig. 8. But, when $(\lambda, p)$ are jointly optimized to minimize the average PAoI, $\lambda=1$ would be optimal. 


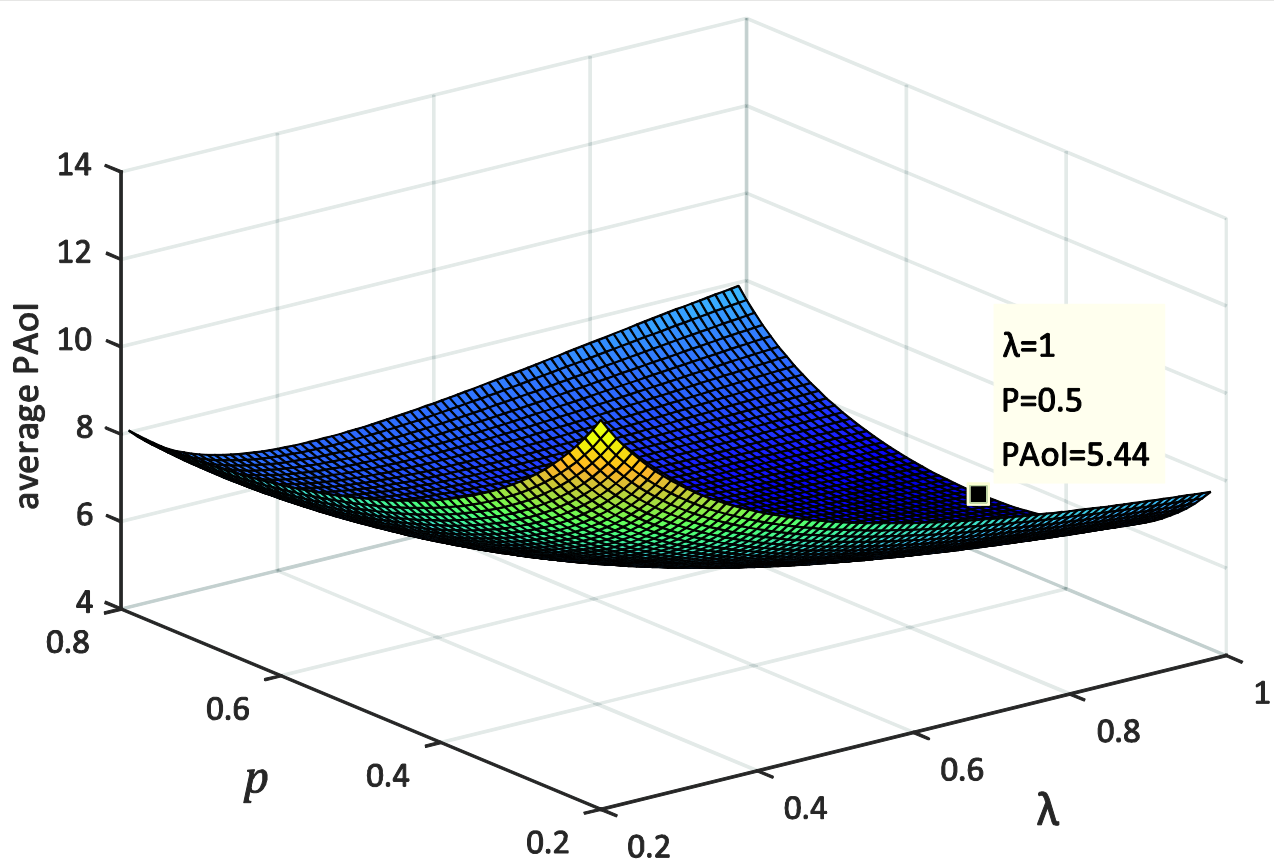

Fig. 8. Average PAoI of symmetric slotted ALOHA networks $((M, q)=(2,0.1))$.

\section{TABLE 1}

FINDING THE OPTIMAL PAOI IN A SYMMETRIC SLOTTED ALOHA NETWORK WITH $q=0.1$.

\begin{tabular}{|c|c|c|c|c|c|}
\hline Number of nodes & 2 & 3 & 4 & 5 & 6 \\
\hline Optimal PAoI & 5.44 & 8.50 & 11.54 & 14.56 & 17.59 \\
\hline Optimal $(\lambda, p)$ & $(1,0.5)$ & $(1,0.33)$ & $(1,0.25)$ & $(1,0.2)$ & $(1,0.17)$ \\
\hline
\end{tabular}

In Fig. 9, we compare the performance of slotted ALOHA network as a decentralized access protocol with the randomized scheduling introduced in [13], as a centralized protocol. In this respect, we consider a symmetric case with $M$ nodes. In the decentralized case, each node transmits based on the same protocol studied in this paper with transmission rate $p$, where value of $p$ has been optimized numerically with the step size of 0.01 . While in the centralized case, a scheduler determines the transmitting node at each slot, randomly. Thus, at each slot, each node might be 


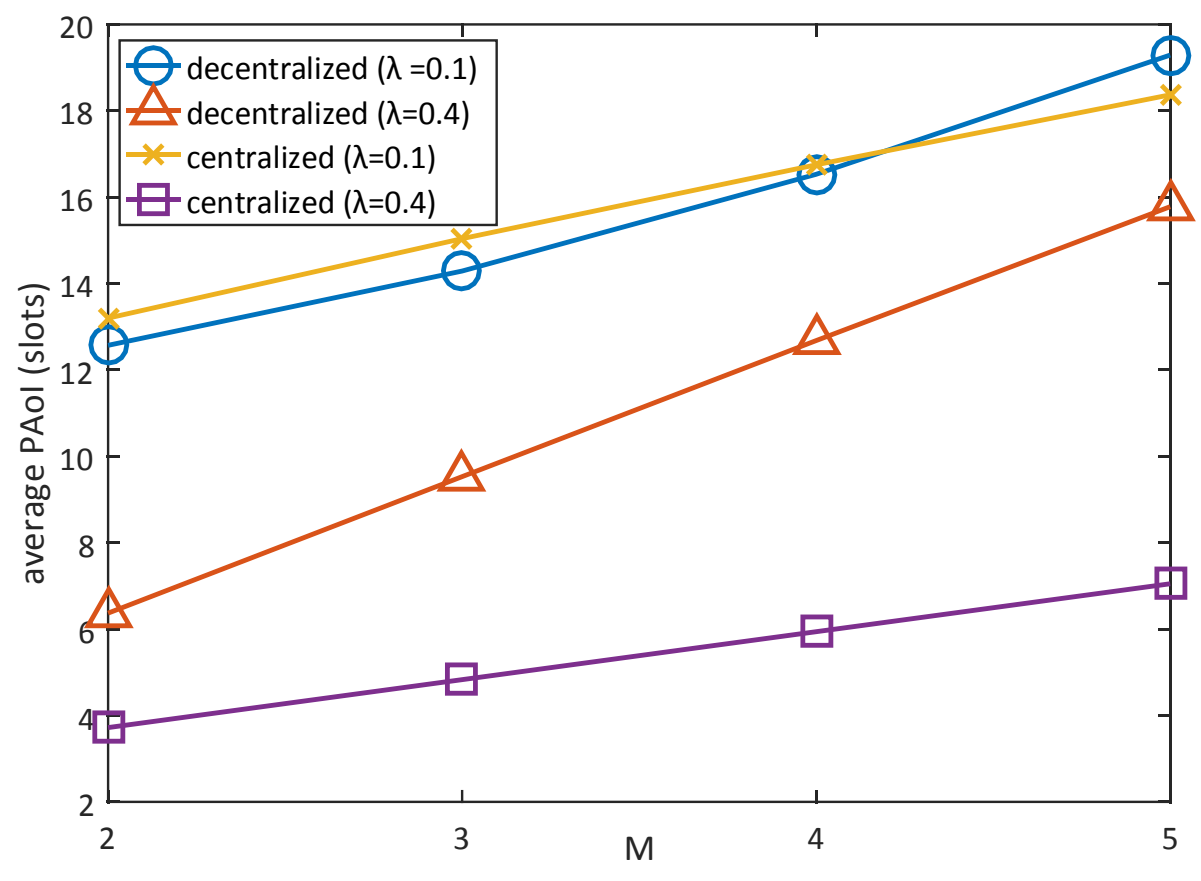

Fig. 9. Comparison of randomized scheduling (centralized) and slotted ALOHA (decentralized) access protocol in a symmetric network.

scheduled to transmit (even if does not have a packet to transmit) with probability $1 / M$ (as explained in [13]). The results show that when the arrival rate is low, slotted ALOHA is preferable while when the arrival rate increases, the randomized scheduling as a centralized protocol is preferable. The reason behind this is that the randomized scheduling protocol introduced in [13], is a non-conservative policy since even a node with an empty server may be scheduled to transmit at a time slot, while slotted ALOHA protocol is a work conserving protocol, because an empty node does not attempt to transmit. But, slotted ALOHA protocol suffers from the probable collisions when at least two nodes transmit at the same time slot. Hence, when the arrival rates are relatively low, the slotted ALOHA protocol is preferable since it is work conservative and the collision probability is low, while when the arrival rates increase and the network becomes more crowded, the centralized policy is preferable as the result of removing collisions.

Next, we consider an asymmetric case again and compare the throughput of our scenario with the traditional slotted ALOHA network where buffer size of each node is infinite and packets of each node are served distinctly. For the sake of simplicity, we consider that $q_{1}=q_{2}=0$. We 


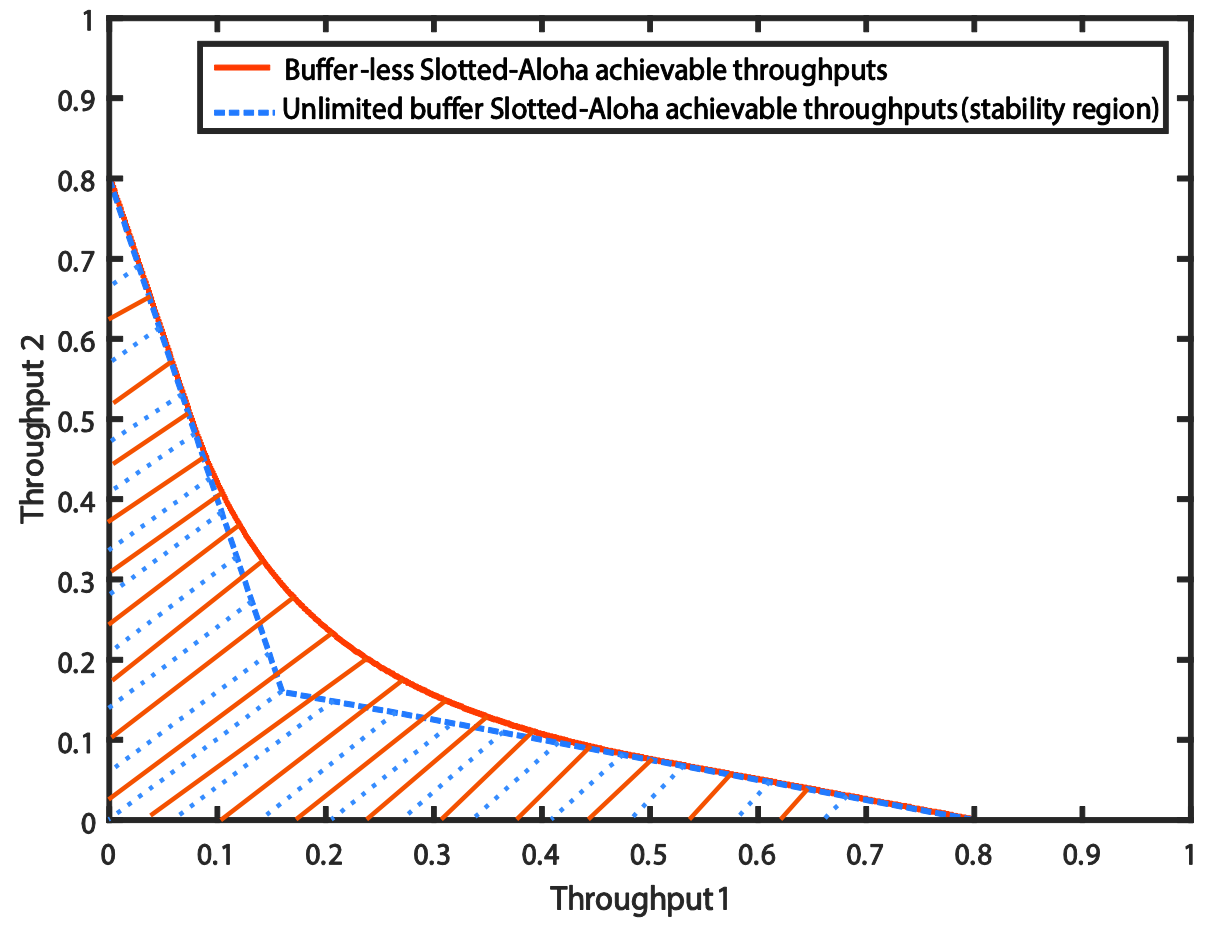

Fig. 10. Achievable throughput region of slotted ALOHA network with unlimited buffer and buffer-less nodes $\left(p_{1}=0.8, p_{2}=0.8\right)$.

focus on the achievable throughput region, i.e., the region including all achievable throughputs of both nodes when the arrival rates of nodes vary from zero to one. The achievable throughput region of traditional slotted ALOHA network is the same as its stability region, which has been evaluated in [27]. But, when the nodes are buffer-less, the throughput of each node could be evaluated from the results of this paper, as aforementioned in Section III, i.e., (5). Fig. 10 shows the achievable throughput region of both networks. We observe that the achievable throughput region of the slotted ALOHA network with buffer-less nodes, includes the achievable throughput region of the network with unlimited buffer nodes. This shows that in the case that traditional slotted ALOHA network is not stable, reducing the buffer size would help to achieve higher throughputs. Thus, according to the results of Fig. 10, when random access protocol is considered, not only bufferless property is superior from AoI point of view, but also could be superior from the achievable throughput point of view. Obviously, more throughput is obtained at the cost of more droppings.

Finally, we investigate the effect of erasure probability on the average PAoI of different nodes, in Fig. 11. To this end, we have increased the erasure probability of node 1 in a network with two 


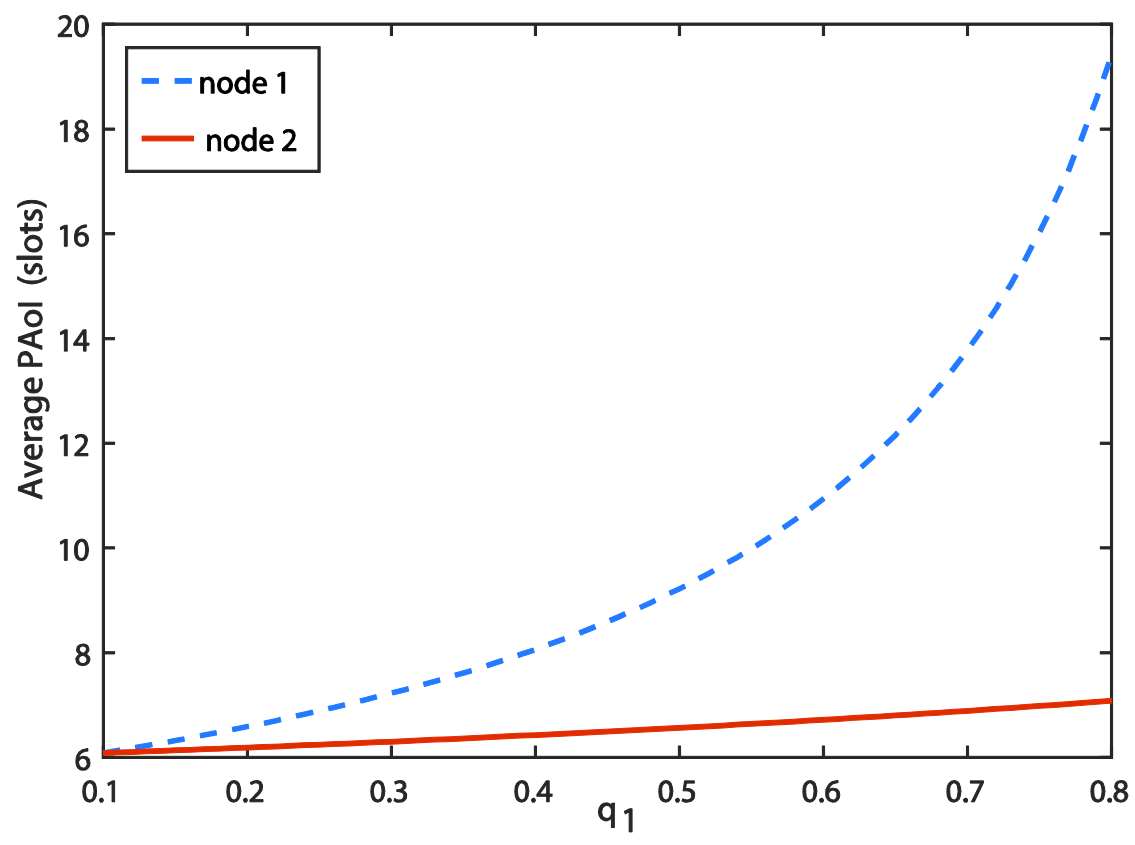

Fig. 11. Effect of erasure probability of node 1 on the PAoI of nodes $\left(\lambda_{1}=\lambda_{2}=0.5, p_{1}=p_{2}=0.65, q_{2}=0.1\right)$.

nodes and studied its effect on the average PAoI of both nodes. As it was expected, average PAoI of both nodes increases. The average PAoI of the first node increases as more transmissions of node 1 become erased at destination. Thus, emptiness probability of node 1 decreases, leading to increase in PAoI of node 2 as a result of increase in collision probability.

\section{CONCLUSION}

In this paper, we studied PAoI in a discrete-time slotted ALOHA network comprised of bufferless nodes. We proposed a trellis-based model to find the average PAoI in a slotted ALOHA network and by using some matrix algebra, we derived the average PAoI in a closed form. Based on our analytical results, we found average PAoI-constrained regions for different values of ARs and TRs. We also numerically derived the optimal values of PAoI in a symmetric slotted ALOHA network and observed that the optimal PAoI is obtained when the arrival rate equals one and transmission probability equals $1 / M$ ( $M$ is the number of nodes). Furthermore, we compared the performance of the slotted ALOHA protocol with a non-conservative randomized scheduling (centralized) access protocol without collision, and concluded that when the ARs are relatively 
low, slotted ALOHA is superior. We Also compared the achievable throughput region of slotted ALOHA network with unlimited buffer and buffer-less nodes. We observed that the achievable throughput region of the buffer-less case includes the achievable throughput region of the unlimited buffer case. At the end, we investigated the effect of increasing erasure probability of a typical node on PAoI of all nodes. We observed that it has a significant effect on the PAoI of the same node and has some degrading effect on PAoI of the other nodes due to increasing collision probability.

\section{REFERENCES}

[1] S. Kaul, M. Gruteser, V. Rai and J. Kenney, "Minimizing age of information in vehicular networks," IEEE Communications Society Conference on Sensor, Mesh and Ad Hoc Communications and Networks, pp. 350-358, 2011.

[2] M. Costa, M. Codreanu, and A. Ephremides, "On the age of information in status update systems with packet management," IEEE Trans. Info Theory, vol. 62, no. 4, pp. 1897-1910, April 2016.

[3] S. Kaul, R. D. Yates, and M. Gruteser, "Real-time status: How often should one update?," in Proc. INFOCOM, 2012.

[4] S. Kaul, R. D. Yates, and M. Gruteser, "Status updates through queues," in Proc. Conf. on Information Sciences and Systems (CISS), March. 2012.

[5] E. Najm and R. Nasser, "The age of information: The gamma awakening," in Proc. IEEE Int'l. Symp. Info. Theory, 2016, pp. 2574-2578.

[6] A.M. Bedewy, Y. Sun, and N.B. Shroff, "Minimizing the age of information through queues," IEEE Trans. Info Theory, vol. 65, no. 8, pp. 5215-5232.

[7] E. Najm, R. Yates \& E. Soljanin, "Status updates through M/G/1/1 queues with HARQ”, in Proc. IEEE ISIT, pp. 131-135, 2017.

[8] S. Asvadi, S. Fardi and F. Ashtiani, "Analysis of Peak Age of Information in Blocking and Preemptive Queueing Policies in a HARQ-Based Wireless Link," IEEE Wireless Communications Letters, vol. 9, no. 9, pp. 1338-1341, 2020.

[9] A. M. Bedewy, Y. Sun and N. B. Shroff. "The age of information in multihop networks." IEEE/ACM Trans on Networking, vol.27, no.3, pp. 1248-1257, 2019. 
[10] A.M. Bedewy, Y. Sun and N.B. Shroff, “Age-optimal information updates in multihop networks,” in Proc. IEEE ISIT, pp. 576-580, 2017.

[11] Y. P. Hsu, E. Modiano, and L. Duan, “Age of information: Design and analysis of optimal scheduling algorithms,” in Proc. IEEE ISIT, pp. 561-565, 2017.

[12] I. Kadota, A. Sinha, E. Uysal-Biyikoglu, Rahul Singh and E. Modiano, "Scheduling Policies for Minimizing Age of Information in Broadcast Wireless Networks," IEEE/ACM Trans. on Networking, vol. 26, no. 6, pp. 26372650, Dec. 2018.

[13] I. Kadota and E. Modiano, "Minimizing the age of information in wireless networks with stochastic arrivals," IEEE Trans. Mobile Computing, 2019.

[14] Z. Jiang, B. Krishnamachari, S. Zhou and Z. Niu, "Can Decentralized Status Update Achieve Universally NearOptimal Age-of-Information in Wireless Multiaccess Channels?" International Teletraffic Congress (ITC 30), pp. 144-152, 2018.

[15] H. Pan and S. C. Liew, "Information Update: TDMA or FDMA?," IEEE Wireless Communications Letters, vol. 9, no. 6, pp. 856-860, 2020.

[16] N. Hirosawa, H. Iimori, G. T. F. de Abreu and K. Ishibashi, "Age-of-Information Minimization in Two-User Multiple Access Channel with Energy Harvesting," in Proc. IEEE 8th International Workshop on Computational Advances in Multi-Sensor Adaptive Processing (CAMSAP), 2019, pp. 361-365.

[17] A. Maatouk, M. Assaad and A. Ephremides, "Minimizing The Age of Information: NOMA or OMA?," in Proc. IEEE INFOCOM, 2019, pp. 102-108.

[18] S. Gopal, S. K. Kaul and R. Chaturvedi, "Coexistence of Age and Throughput Optimizing Networks: A Game Theoretic Approach," in Proc IEEE PIMRC, pp. 1-6, 2019.

[19] R. D. Yates and S. K. Kaul, “Status updates over unreliable multiaccess channels," in Proc. IEEE Int'l. Symp. Info. Theory, pp. 331-335, June, 2017.

[20] H. Chen, Y. Gu, and S.-C. Liew, "Age-of-information dependent random access for massive IoT networks," in Proc. IEEE INFOCOM Workshop on the Age of Information, 2020.

[21]D. C. Atabay, E. Uysal, and O. Kaya, "Improving age of information in random access channels," in Proc. AoI Workshop in conj. with IEEE INFOCOM, pp. 912-917, July 2020.

[22] R. Talak, S. Karaman, and E. Modiano, "Distributed scheduling algorithms for optimizing information freshness in wireless networks," in Proc IEEE SPAWC, pp. 1-5, 2018. 
[23] X. Chen, K. Gatsis, H. Hassani, and SA. Kosta, N. Pappas, A. Ephremides, and V. Angelakis, “Age of information performance of multiaccess strategies with packet management," Journal of Communications and Networks, vol. 21, no. 3, pp. 244-255, 2019.

[24] A. Maatouk, M. Assaad and A. Ephremides, "On the Age of Information in a CSMA Environment," IEEE/ACM Transactions on Networking, vol. 28, no. 2, pp. 818-831, April 2020.

[25] P. S. Dester, P. H. J. Nardelli, F. H. C. d. S. Filho, P. Cardieri and P. Popovski, "Delay and Peak-Age-ofInformation of ALOHA Networks with Limited Retransmissions," accepted in IEEE Wireless Communications Letters.

[26] X. Chen, K. Gatsis, H. Hassani, and S. S. Bidokhti, “Age of information in random access channels," in proc IEEE ISIT, pp. 1770-1775, 2020.

[27] R. Rao and A. Ephremides, "On the stability of interacting queues in a multi-access system,", IEEE Trans. Info Theory, vol. 34, no. 5, pp. $918-930$, Sep. 1988.

[28] W. Szpankowski, "Stability conditions for some multiqueue distributed systems: Buffered random access systems," Advances in Applied Probability, vol. 26, pp. 498-515, 1994.

[29]E. Modiano and A. Ephremides, "A method for delay analysis of interacting queues in multiple access systems," in Proc. Conference on Computer Communications in conj. with IEEE INFOCOM, pp. 447-454, 1993.

[30] R. D. Yates and S. K. Kaul, “The age of information: Real-time status updating by multiple sources," IEEE Trans. Info Theory, vol.65, no. 3, pp. 1807-1827, September. 2018.

[31]H. Takagi, Queueing analysis-Volume 3: Discrete-time systems, Elsevier Science Publisher, 1993. 